\title{
Entgegnung auf die Berichtigung des Herrn Weise in dieser Zeitschrift 1907, 34. (Schrenk oder Schrenck.)
}

\author{
Von Prof. Dr. L. v. Heyden in Bockenheim.
}

In der Wien. E. Z. 1906, 138 steht von mir in Bemerkungen zu Petri's Hyperini der Passus »Die Familie, die ich kenne, schreibt sich Schrenk nicht Schrenck.

Mein lieber alter Freund Weise bemerkt hiezu (siehe Titel) »die älteren russischen Autoren Gebler, Motschulsky etc. schreiben stets richtig Schrenck, wenn sich die Familie jetzt Schrenk nennt, so ist das wahrscheinlich in den russischen Zuständen begründet, wo jeder scheinbar seinen Namen beliebig ändern kann ... «.

Ich habe hierauf zu erwidern, dab ich Leopold v. Schrenk, geboren am 24. April 1826, gestorben am 12. Jänner 1894, persönlich in Nizza 1869 kennen lernte, nachdem meine Familie mit der seinen seit langem bekannt war. Diese nicht russische, sondern baltische Familie schrieb sich stets Sckrenk; die bayerische Familie Freiherr Schrenck von Notzing mit ck. Ich bemerke hiezu, daß ich nicht leichtfertig hinschreibe, was ich nicht verantworten kann.

Freund Weise scheint doch die Literatur nicht so eingehend studiert zu haben, sonst würde er gefunden haben, daß Gebler, den er zuerst zitiert, stets Schrenk schreibt. Ich besitze ein Geblersches Manuskript (1885 von Dr. v. Seidlitz zum Geschenk erhalten) »Verzeichnis der von Herrn Schrenk in der südöstlichen Kirgisensteppe vom Kreise Karkavaly bis an den Fluß Tchu und an die chinesische Grenze gefundenen Käfer«. Hier sind erwähnt:

6. Cicindela Schrenkii m. Bull. Petersbg. 1841.

43. Nebria Schrenkii m. Bull. Petersbg. 1842.

55. Sphodrus Schrenkii m. Bull. Petersbg. 1844.

227. Capnisa Schrenkii m. Bull. Petersbg. 1844.

236. Trigonoscelis Schrenkii m. Bull. Petersbg. 1844.

315. Mylabris Schrenkii m. Bull. Petersbg. 1841.

342. Piazomias Schrenkii Schh. i, l. Bull. Petersbg. (Später schreibt Boheman in Schönherr VIII., II., pg. 410 irrtümlicherweise Schrenchii!)

368. Cleonus Schrenkii m. Bull. Petersbg. 1844.

398. Coniatus (Hyperine!) Schrenkii m. Bull. Petersbg. 1841. 
Es ist ein Auszug aus dem gleichlautenden Verzeichnis im Bull. Mosc. 1859, Nr. II, wo überall nur Schrenk vorkommt und von dem Sekretär der Moskauer Gesellschaft, Dr. Renard, mit Anmerkung versehen ist; auch hier heißt es Schrenk.

Gebler schreibt ferner in Coleopt. spec. nov. a Dr. Schrenk in deserto Kirghis. 1843 detectae Mosquae 1860. Er erwähnt auf pg. 1, 2, 23, 24, 31 den Namen Schrenk und beschreibt pg. 5 Sphodrus Schrenkii, pg. 11 Capnisa Schrenkii, pg. 14 Ocnera Schrenkii, pg. 27 Cleomus Schrenkii.

Faust schreibt, Horae XV, 1881, 147, richtig Coniatus Schrenki Gebl. Wenden wir uns nun zu dem Werke »Reisen und Forschungen im Amurlande« von Dr. Leopold v. Schrenck, Band II, Lfg. I Lepidoptera, Petersburg 1859 von Ménétriés und Lfg. II, Coleoptera, 1860 von Motschulsky bearbeitet, so findet man, daß Ménétriés 114 mal den Namen Schrenck anwendet, 9 Schrenckii-Arten beschreibt, zweimal aber auch Schrenk schreibt, p. 11 M. Leopold Schrenk und M. Schrenk. Motschulsky schreibt immer Schrenck.

Bei Ménétriés kann ich mir diese doppelte Schreibweise nicht leicht erklären; daß Motschulsky anders schreibt wie Gebler wundert mich nicht, haben doch alle seine Arbeiten --alle Achtung vor seiner umfassenden Artkenntnis und seinem scharfen Blick -eine leicht hingeworfene Manier des Schreibens; er kümmerte sich nicht um Äußerlichkeiten, wie ich ihn persönlich 1859 kannte.

Zum Schluß! Die Familie nennt sich nicht »jetzt« Schrenk, sondern schrieb sich stets mit $» \mathbf{k}$. In Rußland kann man auch seinen Namen nicht »beliebig ändern«, wie Freund Weise meint, sondern es lassen sich eben viele russische Worte und Laute nicht einfach in deutschen und romanischen Buchstaben wiedergeben, daher die verschiedenen Schreibweisen von Namen, wie Jak ow leff, S em en ow u. s. w. - Man hat daher bei der Schreibweise Schrenki zu bleiben (wie es Gebler als erster schrieb) so lange nicht das Gegenteil bewiesen wird und dies wird schwer halten. 


\section{$2 \mathrm{BHL}$ Biodiversity Heritage Library}

Heyden, Lucas von. 1907. "Entgegnung auf die Berichtigung des Herrn WEISE in dieser Zeitschrift 1907, 34 (SCHRENK oder SCHRENCK.)." Wiener entomologische Zeitung 26, 77-78. https://doi.org/10.5962/bhl.part.8872.

View This Item Online: https://www.biodiversitylibrary.org/item/45299

DOI: https://doi.org/10.5962/bhl.part.8872

Permalink: https://www.biodiversitylibrary.org/partpdf/8872

\section{Holding Institution}

Smithsonian Libraries

\section{Sponsored by}

Smithsonian

\section{Copyright \& Reuse}

Copyright Status: NOT_IN_COPYRIGHT

This document was created from content at the Biodiversity Heritage Library, the world's largest open access digital library for biodiversity literature and archives. Visit BHL at https://www.biodiversitylibrary.org. 